\title{
Emociones y habilidades comunicativas en la convivencia escolar en la IE Rural del Sur de Tunja
}

\author{
Sandra Patricia Cordero García* \\ Claudia Patricia Chinome Alba*** \\ Andrea del Pilar Garzón Bautista ${ }^{* * * *}$
}

Artículo de reflexión

Fecha de Recepción: 9 diciembre 2017.

Fecha de Aprobación: 30 abril 2018.

\section{Resumen}

La comunidad de la Institución de la influencia de las emociones Educativa Rural del Sur, en Tunja, presenta una problemática relacionada con el maltrato físico y verbal entre estudiantes, acompañado de dificultades en las relaciones personales y rendimiento académico, atribuibles a un contexto social, en el que sus emociones no se desarrollan adecuadamente. Con base en esta situación, este artículo reporta avances de una investigación que se realiza para obtener el título de magister en educación; cuyo objetivo es ofrecer un marco de comprensión y reflexión en el desarrollo de habilidades comunicativas, como parte de una educación integral que garantice una adecuada convivencia escolar. Se propone para esto, el desarrollo de una investigación cualitativa, utilizando estrategias de acción, con base en postulados de Pedagogía del Amor de $\mathrm{H}$. Maturana, haciendo énfasis en el discurso y las experiencias de los estudiantes y docentes en problemas reales relacionados con el contexto cotidiano. El desarrollo del trabajo se resume en cuatro fases: planeación,
* Institución Educativa Rural del Sur - Boyacá Colombia patocg21@yahoo.es ** Institución Educativa Rural del Sur - Boyacá Colombia claudiachinome@hotmail. com

*** Institución Educativa Rural del Sur - Boyacá Colombia andreadelpílar.garzon@ gmail.com

๔) $\Theta \Theta$ 
observación, acción y reflexión. planes de acción y análisis en el vivir y Los resultados se analizan de forma convivir en la escuela. retrospectiva, donde la observación directa, registro de campo, entrevistas a docentes y niños han dado paso a

Palabras Claves: Emociones; convivencia escolar; habilidades comunicativas. 


\section{Introducción}

"Sólo se puede aprender aquello que se ama"

Francisco Mora.

Partiendo del punto de vista nacional, la baja calidad educativa sigue siendo una problemática presente en Colombia, limitando la formación y el desarrollo de competencias para el trabajo y para la vida. La falta de una verdadera educación integral hace que los estudiantes no sólo presenten falencias en materia académica, sino en los diferentes aspectos de su formación, como en competencias ciudadanas, valores éticos y comportamiento en comunidad.

Todo surge de la reflexión y conversación hecha en el segundo semestre del año 2016, donde se muestra una gran preocupación en la Institución Educativa Rural del Sur, puesto que ha venido presentado una serie de problemáticas en materia de convivencia escolar, lo que a su vez afecta directamente el aprendizaje de los estudiantes al no alcanzar los estándares académicos establecidos por el Ministerio de Educación Nacional (MEN), afectando además la comunicación con los docentes y abriendo brechas entre dichas relaciones.

Emociones y habilidades comunicativas en la convivencia escolar, como se denomina el trabajo, se proyecta con la voluntad e interés de los docentes que cursan la Maestría Profundización en Educación, para sacar avante de la pedagogía del amor, postulada por Maturana que busca, "crear las condiciones que lleven al aprendiz a ampliar su capacidad de acción y reflexión en el mundo en que vive, de modo a contribuir para su conservación y transformación de manera responsable, en coherencia con la comunidad y el entorno natural al que pertenece" (Maturana \& Nisis 1997:18), además va acorde al modelo Pedagógico Dialogante seguido por la Institución, buscando un mejor futuro para quienes serán las nuevas generaciones, mediante la ruta de la reflexión y el diálogo, lo que se convierte en el reto como punto de partida de la investigación.

Las directivas y los docentes de la Institución reportan que entre los mismos estudiantes existe violencia física y verbal, consumo de cigarrillos, inasistencia a clases, hurtos, deserción estudiantil y acoso sexual, entre otros. La problemática es creciente y empieza a afectar a los más jóvenes, ya que existe evidencia que los estudiantes de diferentes grados de primaria también son partícipes de peleas dentro y a las afueras del plantel, uso de lenguaje soez, pasando al acoso y abuso físico. Tales comportamientos por parte de los estudiantes se reflejan en las aulas, ya que los docentes afirman que resulta difícil el trabajo en las clases y sus resultados académicos son consecuentes, teniendo bajas calificaciones en la mayoría de asignaturas.

Es importante acotar que estas problemáticas son atribuibles parcial o totalmente a experiencias familiares cercanas que resultan particularmente influyentes en el comportamiento del menor y que en consecuencia
Todo surge de la reflexión y conversación hecha en el segundo semestre del año 2016, donde se muestra una gran preocupación en la Institución Educativa Rural del Sur, puesto que ha venido presentado una serie de problemáticas en materia de convivencia escolar 
afectan su rendimiento académico y su comunicación con el otro, puesto que se encuentra en actitudes defensivas y agresivas.

Estas situaciones problémicas se identificaron como resultado de entrevistas aplicadas a docentes, directivos y encuestas a estudiantes de primaria; de esta manera se consolidando una parte de diagnóstico, que muestra la importancia y necesidad de aprender a escuchar, de dar espacio al dialogo y expresión de emociones para mejorar el ambiente escolar.

Las formas de comunicación se han de propiciar en el aula de clase, que a su vez servirán como objeto de estudio, llevado a través del escrito de diarios de campo. Los procesos comunicativos que el niño exprese a partir del contacto vivencial con otros, permitirán evidenciar la forma en que el estudiante desarrolla una identidad en el ser y el hacer.

La investigación cuya variable o categoría de estudio abarca las emociones, convivencia escolar, habilidades comunicativas, conflicto escolar, espacios de participación, entre otros; llevan a hacer un rastreo en el cual los resultados han demostrado la importancia de reflexionar frente a los procesos socio afectivos, pedagógicos $y$ formativos en el contexto escolar y familiar; además de considerar importante brindar espacios de encuentro que involucren a toda la comunidad educativa de forma agradable, amena y afectuosa, sin olvidar la importancia y relación de la comunicación en este proceso de desarrollo del ser humano.
Lo anteriormente expuesto hace referencia a lo importante de esta investigación, abordaremos este tema considerando aspectos fundamentales como la comunicación en la escuela, que se desarrolla con base en la realidad vivida en el aula, la relación de esta con el contexto y la afectación con el ser y hacer de los niños. En segundo lugar, se establece antecedentes de campo que muestran la realidad expuesta y las miradas desde otras visiones de autores que se relacionan con la investigación; en tercer lugar, presentamos la metodología desarrollada, la investigación acción sustenta el proyecto y presenta resultados parciales de dos de las cuatro fases; donde se evidencian la importancia de la problemática abordada. En cuarto lugar, se presentan algunas reflexiones hechas hasta este momento de las acciones propuestas.

\section{Comunicarse en la Escuela y en el Aula}

Para abordar nuestra problemática es importante acotar algunas de las reflexiones y preguntas que han surgido a través de este proceso, además de reconocer la importancia del contexto en el cual trabajamos y la relación de investigaciones realizadas con la problemática expuesta. Este es el punto de partida y la base que sustenta el trabajo.

"En el conversar construimos nuestra realidad con el otro. No es una cosa abstracta. El conversar es un modo particular de vivir juntos en coordinaciones del hacer y del emocionar. Por eso el conversar es constructor de 
realidades. Al operar en el lenguaje cambia nuestra fisiología. Por eso nos podemos herir o acariciar con las palabras. En este espacio relacional uno puede vivir en la exigencia o en la armonía con los otros. O se vive en el bienestar estético de una convivencia armónica, o en el sufrimiento de la exigencia negadora continua”. (Maturana, 2008 p. 23)

La situación social y educativa en Colombia nos muestra que no es suficiente con obtener puntajes altos en asignaturas que demuestran competencias cognitivas, la forma como se construye cada ser humano y las relaciones que establece también deben ser objeto de atención y trabajo en la escuela, considerando especialmente que vivimos un momento donde el pos-conflicto es un tema social recurrente, exige comprender la forma como tenemos que prepararnos para comprender la violencia que vive el país, la situación de cientos de niños que no tienen oportunidades de establecer relaciones afectivas que los ayuden a construirse personal y socialmente. La situación de padres y familias enteras que han sido objeto de mal trato y menosprecio por la situación de orden social y político que por décadas ha vivido nuestro país.

¿Cómo se prepara el maestro para abordar esta situación? La respuesta no es fácil porque hace tiempos que la violencia y el maltrato está presente en la escuela colombiana, podría decirse que es una situación que tiende a normalizarse, pero con el tiempo ha llegado a unos niveles insoportables para el clima escolar y el aula en especial, en la escuela se aprende y se enseña en espacios que necesariamente requieren convivir.

La Institución Educativa Rural del Sur de la ciudad de Tunja (Boyacá), cuenta con siete sedes y se caracteriza por atender población urbana marginal, campesina y desplazada, con problemas de descomposición familiar, madres solteras cabeza de familia, promiscuidad, consumo de bebidas alcohólicas, maltrato intrafamiliar, familias monoparentales y extendidas que generan conflictos de alto riesgo psicosocial. La mayoría de los padres cuentan con una formación de básica primaria, quienes algunas veces manifiestan poco aprecio por el estudio, impactando negativamente en los hijos. Sin embargo, se ha ido ampliando la perspectiva de crecimiento personal y familiar a través de nuevos programas de educación para adultos.

El sector donde se encuentra ubicada la Institución Educativa se dedica a la agricultura en pequeñas parcelas (minifundios), en un sistema de jornaleros y arrendatarios, complementada con la atención de pequeños hatos. Un buen número de familias deriva su sustento del mercado de productos agrícolas en la plaza del sur de la ciudad, otro grupo lo logra del transporte en calidad de conductores, mientras que otros son propietarios de pequeños restaurantes dedicados a la producción y comercialización del cerdo. No obstante, el trabajo informal es preponderante, haciendo que la estabilidad económica de muchas de las familias del sector sea baja.
La Institución Educativa Rural del Sur de la ciudad de Tunja (Boyacá), cuenta con siete sedes y se caracteriza por atender población urbana marginal, campesina y desplazada, con problemas de descomposición familiar, madres solteras cabeza de familia, promiscuidad, consumo de bebidas alcohólicas, maltrato intrafamiliar, familias monoparentales y extendidas que generan conflictos de alto riesgo psicosocial. 
La situación geomorfológica del sector, las características psicosociales en la conformación de las familias, el grado de escolaridad y las actividades de las cuales se obtienen los recursos económicos para la manutención, son ambientes que motivan la investigación hacia la mitigación de posibles conflictos, en aras de encontrar en la comunicación adecuada el manejo de las emociones y la práctica de la tolerancia entre todos los miembros de la Comunidad Educativa.

\section{Relación del Proyecto con otros estudios.}

Reconociendo aspectos más específicos del sector, nos es indispensable hacer un rastreo de estudios relacionados con las emociones y su reconocimiento en el ámbito escolar, las habilidades comunicativas y su incidencia en los procesos de crecimiento y relación social, teniendo en cuenta su metodología y resultados obtenidos.

Se realizó una búsqueda de bibliografía existente en la Universidad Pedagógica y Tecnológica de Colombia que permitiera dar cuenta de la metodología utilizada en los proyectos de grado o tesis realizadas por estudiantes de la Universidad. En el catálogo de proyectos de grado UPTC, existen dieciséis (16) resultados utilizando como palabra clave de búsqueda "convivencia escolar”. De éstos, apenas uno (1) es un trabajo reciente, ya que el resto son anteriores a 2010. Los estudios abarcan relaciones entre la convivencia escolar con la responsabilidad, la inteligencia emocional y el maltrato familiar; también se encuentran algunos ejemplos de usos de herramientas pedagógicas centradas en la educación física, la lúdica y el fortalecimiento de la autoestima como estrategias de mejoramiento de la convivencia escolar.

Siguiendo el mismo procedimiento, pero utilizando "habilidades comunicativas" como palabra clave, se encontraron cuarenta y cinco (45) resultados, todos posteriores a 2010 que abordan de diversas maneras y con diferentes estrategias el fomento de las habilidades comunicativas en los alumnos como meta final.

De ahí la importancia de hacer de esta iniciativa una investigación innovadora en la UPTC y la Institución Educativa Rural del Sur, porque reconociendo que la emocionalidad de los niños y los maestros también hacen parte de la escuela, tradicionalmente se ha dado toda la importancia a la información, descuidando el sentir y pensar de los niños, se cree que la información se adquiere muy fácilmente hoy en día a través de las Tecnologías de la Información y la Comunicación (TIC), pero la convivencia se construye en el día a día y determina la forma como los niños aprenden a actuar en la sociedad en que viven.

Para la Institución Educativa Rural del Sur, los niños son el alma y la lucha de mejora diaria, sus familias el sustento y los docentes los promotores del cambio, por tal razón la formación integral apoyada por valores, principios, convivencia, comunicación, emociones, respeto, 
Derechos Humanos y tolerancia serán los pilares primordiales para el éxito de la transformación que se propone en el entorno en que se vive.

\section{Antecedentes de Campo}

Aquí se muestra la realidad expuesta y las miradas desde otras visiones de autores que se relacionan con la investigación, además de estar clasificadas teniendo en cuenta las temáticas relacionadas con la investigación, clima escolar, competencias socioafectivas, habilidades comunicativas, emociones, desarrollo emocional, expresión y manifestación emocional, expresión social y familiar. Es tan solo una parte del acercamiento y relación de diferentes investigaciones y su aporte a nuestro trabajo

\section{Clima Escolar}

El clima escolar ha sido objeto de estudio en muchas oportunidades destacándose la importancia de procurar unas relaciones de aceptación entre pares y reconocimiento de las diferencias como parte del proceso educativo y por lo tanto de formación integral de los estudiantes, en este proceso además es indispensable involucrar a toda la comunidad educativa, dando oportunidad de participación (Buitrago \& Cardenas, 2017).

Enrrique Chaux es un psicólogo colombiano que ha dedicado gran parte de sus investigaciones a estudiar dificultades de convivencia en la escuela, competencias ciudadanas y acosos escolar. En su libro "Educación, convivencia y agresión escolar” (2002) muestra de manera sencilla el producto de sus investigaciones y la reflexión frente a estas, ha sido un sustento importante por sus aportes en el contexto educativo colombiano.

Algunos artículos, tesis, libros referentes al tema se nombran a continuación y son valiosos clasificarlos según las categorías:

Un primer trabajo corresponde a Castro y Gaviria (2005), llamado Clima escolar y comportamientos psicosociales en niños, y exploró la asociación entre los comportamientos psicosociales de los niños y el clima escolar, medido como las relaciones entre pares en el aula. Se realizó un estudio de corte transversal de fuentes secundarias de una muestra de 631 niños de 6 establecimientos públicos y privados de estrato dos, de la ciudad de Medellín en el año 2001. Se aplicaron pruebas socio métricas para evaluar los comportamientos de la relación social existente entre los niños pertenecientes a cada uno de los grupos. El 23,8\% de los niños entre los 3 y 11 años presentaron al menos un síntoma de problemas psicosociales, siendo los más frecuentes la depresiónansiedad y la agresividad indirecta.

Esta investigación concluye que el desempeño académico es una variable de predicción de la aceptación de un niño en el aula. La exclusión social en el aula conlleva a afectar su salud mental y sus relaciones en los ámbitos escolar, familiar y social. Para nuestro proyecto es importante e interesante ver estudios que se hacen en pro de conocer y analizar concienzudamente la relación existente dentro del aula, espacio fundamental en las relaciones
Enrrique Chaux es un psicólogo colombiano que ha dedicado gran parte de sus investigaciones a estudiar dificultades de convivencia en la escuela, competencias ciudadanas y acosos escolar. 


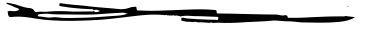

Rodríguez (2010) en su artículo "La oralidad, la lectura y la escritura como mediaciones para la convivencia", hace referencia a la convivencia como tarea personal y colectiva. humanas y donde las emociones pueden ser factor primordial en estas.

Salazar y Lara-Salcedo (2008) en su trabajo "La mediación del conflicto escolar a la construcción de comunidades justas", explican que el estudio pretende enriquecer el campo de conocimiento y discusión sobre la convivencia escolar. Este artículo se dio a raíz de un encuentro Nacional sobre Modelos y experiencias de mediación, donde se seleccionaron dos experiencias innovadoras de Bogotá y una de Medellín. Su metodología se dividió en tres momentos uno de análisis de experiencias, otro de contacto con las instituciones donde se desarrollaron entrevistas a estudiantes y docentes; el tercer momento se organizó, analizo y se presentó un informe de socialización de resultados. Como conclusiones resaltan la importancia de la democracia en la vida escolar, el fortalecimiento de los lazos entre estudiante - docente, la importancia del desarrollo de acciones donde nos reconozcamos como actores sociales, la construcción y el fortalecimiento de un sentido de comunidad, entre otras.

Otro aporte importante para el proyecto es la investigación de Mendoza y RicoBallesteros (2014), donde se explica el clima escolar como elemento fundamental de la convivencia en la escuela. Su muestra fueron 152 estudiantes, docentes y directivos y tuvo como objetivo caracterizar el clima escolar en instituciones educativas de Barranquilla, se aplicó un protocolo para la medición de convivencia. La información obtenida señala que producto de la interacción de los estudiantes se generan escenarios de irrespeto, ofensas verbales, apodos, burlas, etc., que conllevan a conflictos escolares. Un aporte valioso es la importancia que los estudiantes le dan a la escuela como lugar de compartir entre pares, algunos dicen que es un lugar de tranquilidad; estas respuestas son parte importante de una reflexión continua hacia la investigación.

Rodríguez (2010) en su artículo " $\mathrm{La}$ oralidad, la lectura y la escritura como mediaciones para la convivencia”, hace referencia a la convivencia como tarea personal y colectiva. El autor presenta reflexiones y estrategias encaminadas a fomentarla y desarrollarla en la escuela. Así mismo, escribe que la convivencia no es un estado ni una condición espontánea y que las habilidades comunicativas son acciones que se construyen con el tiempo y son mediadoras de convivencia.

\section{Competencias ciudadanas}

Se hace referencia al desarrollo de competencias a través de aprendizajes que permitan a los niños y las niñas, por medio de las interacciones sociales apropiarse de su realidad física $y$ social; convirtiéndose en medio para expresar sentimientos, pensamientos, ideas, emociones, que servirán como fundamento para propiciar ambientes de aprendizaje significativo en donde las relaciones se basen en el respeto, la tolerancia y con ello garantizar mejores desempeños en el contexto escolar, comunitario y sociocultural de los niños y niñas. 
Es necesario para el fortalecimiento de las competencias ciudadanas tener en cuenta el desarrollo de las capacidades cognitivas, emocionalesycomunicativas que integradas entre sí y relacionadas con conocimientos y actitudes, hacen posible que el ciudadano actúe de manera constructiva en la sociedad (Ruiz Silva \& Chaux Torres, 2005).

En Colombia se tuvo en cuenta referente la Ley general de educación de Colombia, (Ley 115, 1995) que define al proceso educativo como "una formación permanente, personal, cultural y social que se fundamenta en una concepción integral de la persona humana, de su dignidad de sus derechos y deberes". De igual forma se tuvo en cuenta el Articulo 5, numeral 1 , donde se menciona uno de los fines de la educación: "el pleno desarrollo de la personalidad, sin más limitaciones que las que te imponen los derechos de los demás y el orden jurídico, dentro de un proceso de formación integral, física, psíquica, intelectual, moral, espiritual, social, afectiva, ética, cívica y demás valores humanos”.

Es de vital importancia que los niños y niñas desarrollen en un alto grado las competencias ciudadanas que le permitan acoplarse en forma óptima a la sociedad, convirtiéndose en actores importantes en la construcción y desarrollo de la misma.

\section{Competencias Socioafectivas}

En el año 2006 el Ministerio de Educación Nacional (MEN) establece lineamientos para el desarrollo de competencias básicas, entre ella la competencia ciudadana dónde se manifiesta la responsabilidad de la escuela de formar individuos para la convivencia, pueden ser los primeros pasos para pensar que no solo las competencias matemáticas o del lenguaje entre otras, son indispensables en la escuela, a parir de esta idea surge con mayor precisión el tema de la convivencia, investigadores como Miguel de Subiría Samper(2009) quien plantea la competencia socio-afectiva, refiriéndose a la capacidad del individuo de reconocerse a sí mismo generando autonomía, una forma de valorarse y valorar a otros y pensar la construcción de relaciones efectivas, un tema que debe ser responsabilidad de la escuela, así como se tiene la responsabilidad de formar individuos en lo cognitivo también está la responsabilidad de lo ético.

Serna (2014), en el artículo "Competencia Socio Afectiva en el Marco Escolar Colombiano", da a conocer los resultados de una investigación donde la muestra fueron 64 estudiantes de grado $5^{\circ}$ de primaria de la ciudad de Barranquilla. La estrategia de implementación consistió en aplicación de encuestas, observaciones, revisión documental; desde un modelo dialogante que se basa en el trabajo en aprendizajes cooperativos. Su aporte es muy valioso para nuestro proyecto cuando hablamos de la comunicación y el lenguaje como mediadores en el desarrollo de competencias Socioafectivas.

García-Sánchez y Ortiz-Molina (2012) en su libro Los maestros ante la violencia
Serna (2014), en el artículo "Competencia Socio Afectiva en el Marco Escolar Colombiano", da a conocer los resultados de una investigación donde la muestra fueron 64 estudiantes de grado $5^{\circ}$ de primaria de la ciudad de Barranquilla. 
escolar, hacen referencia a conceptos relacionados con nuestra propuesta de estudio, hablan de las relaciones socioafectivas y cómo estas influyen en los estudiantes construyendo relaciones más fuertes y aprendizajes más significativos. En la lectura retoman postulados de Torres (2006, pp. 85) que afirman que durante mucho tiempo en el mundo de la educación sólo se prestaba atención explícita a las dimensiones intelectuales, olvidando que los sentimientos afectos y expectativas positivas son uno de los motores más importantes para movilizar e implicar a las personas.

\section{Habilidades comunicativas}

Para Maturana (2001) las palabras se refieren a entidades configuradas en la afluencia de las coordinaciones de acciones humanas consensuales que podemos referir cuando operamos en el lenguaje. Lo mismo ocurre con la concepción de 'mente', en la que se asume que la mente no puede existir sin lenguaje, ni la reflexión tampoco.

Según este planteamiento, podemos deducir que el ser humano para comunicarse utiliza no solamente la expresión verbal, ya que a través de todo su ser puede comunicarse o hacerse entender de sus semejantes. Es decir que el sujeto no necesariamente necesita valerse del habla para comunicarse, sino que de acuerdo a las circunstancias usa los recursos que en la inmediatez le permitan "lenguajear" con sus pares.

En cuanto al lenguaje no verbal podemos concluir que los seres humanos sin darnos cuenta emitimos mensajes del estado de ánimo y carácter a través de los movimientos. El cuerpo comunica sin palabras mediante la expresión facial, mirada, sonrisa o ausencia de ella, posición del tronco y extremidades. Por esta razón, cuando un docente se encuentra en un aula de clase sus movimientos corporales y uso del espacio comunican a los estudiantes su ánimo, actitudes y emociones (Maturana, 2001).

El artículo "Competencias Ciudadanas encaminadas al desarrollo de habilidades comunicativas en los estudiantes de grado 4" de Buitrago \& Castro (2005), es un trabajo de Tesis desarrollado en el municipio de Chiquinquirá (Boyacá), que, a través de observaciones directas y participativas, de encuestas aplicadas, tabuladas y analizadas se determina la necesidad de abordar las competencia ciudadanas dentro del aula y de elaborar estrategias dinámicas y comprometidas a favorecer el diario quehacer educativo y la oportunidad para establecer la armonía en la convivencia como camino a la felicidad. Las producciones escritas se dan como medio de expresión de la realidad social de un grupo.

Duarte (2005) en el artículo "Comunicación y convivencia escolar en la ciudad de Medellín, Colombia", hace referencia a la importancia de la comunicación y su relación con la convivencia escolar, en esta ciudad se han venido apropiando con el tema de convivencia escolar y generando reflexión constante de esta y la necesidad de brindar espacios donde la comunicación permita el 
reconocimiento de sí mismo y del otro, y que es a través de ella desde donde podrán desplegarse estrategias, iniciativas y pautas creativas con respecto a la convivencia ciudadana.

En el proyecto de grado "Talleres de argumentación: una berramienta para mejorar el nivel de convivencia en los estudiantes de grado 9 del centro Juvenil Emiliani de la ciudad de Tunja", Cely (2010) utilizó una metodología activa donde los Talleres de Argumentación fuesen el medio para propiciar conciencia en cuanto a la incapacidad de dar una buena resolución a los conflictos del aula.

\section{Emociones}

En su libro El Sentido de lo Humano, Humberto Maturana (2002) hace una sentida reflexión de la pedagogía del amor, de la importancia del lenguaje y las emociones en la construcción de actitudes, de las emociones como fundamento social, del papel del docente en la inminente causa - efecto que comparte en su diario vivir con los estudiantes; todo esto desde la biología del ser humano. Apasionante y muy reflexivo este libro y sobre todo muy asertivo como fundamento de nuestro proyecto, en cuanto a la importancia de ver más allá de lo aparente, de hacer más interesante el papel del docente.

Siguiendo con Maturana, el autor habla sobre la afectividad como una ramificación del amor afirmando que los seres humanos -en sentido estrictoflorecemos del amor, porque el amor como emoción constituye el dominio de acciones de aceptación recíproca en el que pudo surgir y conservarse el lenguaje, añadiéndose al modo de vida de nuestros ancestros homínidos como parte constitutiva del vivir que nos define.

De acuerdo con lo anterior se puede decir que la afectividad en los seres humanos está implícita en cada uno y que las emociones son parte fundamental del desarrollo de las personas. Debemos preocuparnos por recobrar la capacidad de integrar los sentimientos en el desarrollo social de los seres humanos, recordando que la familia es el primer lugar donde los niños y niñas por medio de la interacción con sus padres aprenden a manejar las emociones de manera apropiada o equivocada.

Podemos centrarnos en una sentida reflexión de la pedagogía del amor, de la importancia del lenguaje y las emociones en la construcción de actitudes, de las emociones como fundamento social, del papel del docente en la inminente causa - efecto que comparte en su diario vivir con los estudiantes; tomando como punto de partida la biología del ser humano. Apasionantey muy reflexivo el tejido que construye Humberto Maturana entre emociones, lenguaje, y comunicación para presentar la pedagogía del amor como fundamento para pensar nuestra práctica pedagógica.

Vissupe, Angulo-Gallo y Guerra Morales (2017) en su artículo "Características de la expresión emocional en los escolares primarios y su manejo desde la
Podemos centrarnos en una sentida reflexión de la pedagogía del amor, de la importancia del lenguaje y las emociones en la construcción de actitudes, de las emociones como fundamento social 


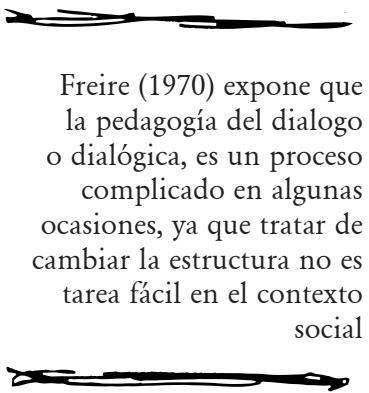

Freire (1970) expone que a pedagogía del dialogo complicado en algunas cambiar la estructura no es tarea fácil en el contexto social perspectiva del personal docente-educativo", tuvieron como población tres centros educativos rurales de primaria en la provincia de Villa Clara Cuba y con la participación de 53 docentes. El objeto de estudio fue el caracterizar las emociones que se manejan dentro del aula de clase y como el docente las identifica y las maneja. Los resultados muestran que los docentes identifican algunas emociones en los estudiantes, pero no cuentan con la capacitación y metodología para manejarlas, también deja la reflexión de continuar con el estudio de las emociones infantiles y de la importancia de estas en el desarrollo social y afectivo de los niños.

\section{Desarrollo emocional}

El desarrollo emocional en los niños y niñas está compuesto por varios elementos, relacionados algunos con comportamientos innatos y aspectos biológicos que son modificados de acuerdo con sus necesidades y el contexto social donde se desarrollan.

Freire (1970) expone que la pedagogía del dialogo o dialógica, es un proceso complicado en algunas ocasiones, ya que tratar de cambiar la estructura no es tarea fácil en el contexto social, pero es necesario e importante hacer ver la importancia de la responsabilidad al igual que hay que señalar los limites, pues no hay vida y libertad sin límites.

El desarrollo emocional es un complemento del desarrollo cognitivo constituyéndose en un elemento decisivo en el desarrollo de la personalidad integral. En nuestro proyecto se quiere desarrollar talleres sobre el buen manejo de las emociones, para que les sea más fácil a los niños y niñas reaccionar frente a las situaciones del diario vivir. Esto permite mejorar la comunicación entre pares y por ende la convivencia en el entorno social de la Institución Educativa Rural del Sur, sede José Joaquín Castro Martínez con los estudiantes de $3^{\circ} \mathrm{A}$.

Goleman (1997) propone como posible solución a dicha situación "forjar una nueva visión acerca del papel que deben desempeñar las escuelas en la educación integral del estudiante, reconociendo en las aulas emoción y cognición. En tal sentido, la educación debe incluir en sus programas la enseñanza de habilidades $\tan$ esencialmente humanas como el autoconocimiento, el autocontrol, la empatía, y el arte de escuchar, así como resolver conflictos y la colaboración con los demás”.

\section{Expresión y manifestación emocional}

La expresión emocional aprueba un acercamiento clave a las emociones que pueda procesar un individuo, permitiendo realizar una interpretación del contexto social, la practica en el ámbito educativo, busca la formación y potenciación de sujetos que sean capaces de expresar, emociones y actuar en el dialogo negociador con los otros.

Freire (1993) señala que el individuo no piensa auténticamente si los otros no piensan también. El carácter dialógico implícito en la afirmación representa la condición fundamental de que el acto de conocer se conjuga de forma paralela con la relación dialógica entre individuos. 
Es sabido también, que es necesario realimentar constantemente las emociones, ya que éstas juegan un papel fundamental en el desarrollo de los niños y niñas, permitiéndoles mejorar en aspectos como el rendimiento académico, el aprendizaje colaborativo, la adaptación escolar la toma de decisiones, la salud, la resolución de conflictos y el liderazgo a lo largo de la vida.

\section{Expresión social y familiar}

Es a partir de la convivencia en familia y la sociedad que las dimensiones del ser y del hacer se van moldeando mutuamente, donde juegan un papel importante las emociones influyendo en las acciones, comportamientos y conductas de los niños y niñas.

Son las emociones, según Maturana (1999), que moldean el operar de la inteligencia y abren y cierran los caminos para posibles consensos a ser establecidos en nuestra vida cotidiana. Él ejemplifica, diciendo que la envidia, el miedo, la ambición y la competición restringen la conducta inteligente porque estrechan la visión y la atención. Para él, solamente el amor amplía la visión en la aceptación de sí mismo y del otro, a partir de las condiciones en que se vive y expanden las posibilidades de un operar más inteligente.

Es en el proceso de transformación en la convivencia que el ser humano se conserva, o no, en su humanidad. Lo mismo ocurre durante el proceso educacional, donde nos transformamos espontáneamente, congruentes con la transformación del otro en el espacio de convivencia. Lo que nos hace humano, según este punto de vista, es nuestro vivir como seres "linguajantes", cooperativos y amorosos, con conciencia de sí mismo y con conciencia social, en el respeto por sí mismo y por los otros (Maturana, 1999). Para Maturana \& Nisis (1997), nuestra humanidad no estaría asociada solamente a nuestra dimensión constitucional, sino también a nuestra manera de vivir/convivir. Es esto que determinaría, en gran parte, nuestro caminar presente y futuro.

\section{Metodología}

Hace referencia a los autores y sus aportes para el desarrollo de la investigación, dando a conocer la estructura y el avance realizado hasta ahora, para el logro de los objetivos planteados.

Se tiene en cuenta el tipo de investigación, enfoque y método, que según el autor y de acuerdo a las necesidades del contexto, establece la ruta metodológica que será el eje del proyecto.

El trabajo se desarrolla teniendo en cuenta el método de investigación cualitativa, con enfoque critico - social y tipo de Investigación - Acción, los participantes son los estudiantes de grado tercero de primaria de la Institución Educativa Rural del Sur, sede José Joaquín Castro Martínez. A este grupo pertenecen un total de 20 hombres y 16 mujeres con edades que oscilan entre los 8 y los 10 años.

Los instrumentos usados están implementados en pro del reconocimiento y cumplimiento de lo 
propuesto Kemmis y McTaggart (1992) que tiene como propósito el cambio educativo a través de las prácticas diarias de los docentes, mediante el conocimiento, comprensión, la transformación practica y la reflexión continúa de nuestro quehacer. Se hace importante el trabajo colaborativo, somos seres únicos, pero vivimos en relación con el otro, de ahí la importancia de nuestra labor diaria, es necesario partir de la observación continua, llevando a cabo las cuatro etapas propuestas por la teoría: planificación, acción-reflexión, observación y evaluación. El proceso que lleva la investigación nos permite dar cuenta de las dos primeras fases, por lo tanto haremos referencia a ellas:

La planificación, es el punto de partida para dar reconocimiento al contexto, reflexionando sobre las experiencias diarias e identificando la problemática que más preocupa. Es fundamental referenciar la problemática para profundizarla y llevarla a la práctica. Es un proceso fundamental que va enriqueciendo con el tiempo y que requiere de un compromiso serio y ético a la hora de ejecutarlo. En esta etapa se elabora un diagnóstico para caracterizar la población objeto de estudio. Se hace una selección de textos y autores para conceptualizar sobre las emociones, la comunicación y convivencia de los niños, haciendo un barrido de bibliografía para el abordaje y apropiación de nuestros temas de estudio.

La acción, es la puesta en marcha de la praxis que cumple un papel importante, donde se reconoce la naturaleza de la problemática de forma real, aquí se observan las relaciones sociales, el lenguaje, trabajo en equipo, mejora actitudes y situaciones del aula.

Aquí se nutre la teoría con la práctica, llega la ejecución para cumplir los propósitos a través de las estrategias propuestas, teniendo claro los instrumentos. Hay que tener en cuenta el trabajo con docentes y estudiantes. Se propiciarán espacios para el compartir de los niños, aplicación de talleres, entrevistas, encuestas, conversatorios, autobiografías de los niños, elaboración de portafolios.

\section{Reflexiones}

Se hace un llamado constante a educar y aprender desde la pedagogía del amor y el respeto, buscar que los niños quieran su escuela y amen estudiar, a través de las emociones.

Es importante acotar que la apropiación teórica de las investigaciones brinda un sustento fundamental en el desarrollo y aplicación del trabajo.

La sistematización de experiencias del diario de campo ha hecho más significativa la reflexión y perspectiva del quehacer docente dentro del aula, además que muestra actitudes notorias de la influencia de las emociones en el comportamiento de los estudiantes y docentes.

Este trabajo brinda una valiosa oportunidad de evidenciar que educar no es solo transmitir una serie de contenidos, educar es sentir, pero sobre todo darse la oportunidad de conocer las necesidades de otros, para ello se debe dar ese espacio de conversar con 
estudiantes, de escucharlos; de sentir sus emociones y aprender a conocer su mundo, que en este contexto en ocasiones se torna cruel.

La investigación resulta una vivencia enriquecedora y única para los docentes investigadores, ya que les permite desarrollar nuevas estrategias comunicativas y pedagógicas que conlleven a un acercamiento a las realidades de los niños, donde el amor y compromiso sean el motor de cambio para la comunidad.

Debemos humanizar la educación, devolver la alegría pérdida a nuestros niños, contamos hoy en día con generaciones tristes y deprimidas, la forma de hacerlo es transmitiendo nuestro cambio interior con amor, con alegría, pero sobre todo con pasión por la educación, que nos permite hacer cambios importantes y trascendentes.

Así, esta es la oportunidad para invitar a otros compañeros docentes, estudiantes y comunidad en general para que tengan en cuenta en sus prácticas pedagógicas la dimensión emocional, la comunicación asertiva y la convivencia ya que éstas son parte fundamental de los seres humanos.

Se tiene en las manos la responsabilidad de contribuir en la formación de las futuras generaciones, el reto está en generar cambios positivos dentro de las comunidades en las que se trabaja y así poder formar no solo seres intelectuales sino personas sensibles para que enfrenten las diferentes situaciones que se les presente.

Son muchos los interrogantes que se pueden develar de esta investigación, pero las más relevantes serian: ¿Como los docentes con sus emociones afectan el convivir en el aula?

¿Hasta dónde los docentes estamos en la capacidad de redefinir conductas y apropiaciones de la realidad vivida en el contexto?

\section{Referencias}

Betancourt, C. M. (8 de junio de 2016). Hallan Síndrome sin cura en Boyacá. El Tiempo. Recuperado de http://www.eltiempo.com/archivo/documento/ CMS-16613976.

Bogdan., S. T. (2000). Introducción a métodos cualitativos de investigación. Barcelona: Paidós.

Buitrago, M. Y., \& Castro, M. H. (2005). Competencias Ciudadanas encaminadas al desarrollo de habilidades comunicativas en los estudiantes de grado 4. (Tesis de maestría), Universidad Pedagógica y Tecnológica de Colombia, Tunja.

Buitrago, R., \& Cárdenas, R. (2017). Emociones e identidad profesional docente: relaciones e incidencia. Praxis \& Saber, 8(17), 225 - $247 . \quad$ https://doi. org/10.19053/22160159.v8.n17.2018.7208

Para el desarrollo de este taller, una de las actividades realizadas consistió en dar una lista de palabras a los estudiantes, ellos debían señalar de la lista dada, cuáles eran inventadas, o reconocidas como pseudopalabras, esto con el fin de identificar el grado de decisión léxica de cada estudiante 
Cely, A. Y. (2010). Talleres de argumentación: una herramienta para mejorar el nivel de convivencia en los estudiantes de grado 9 del centro Juvenil Emiliani de la ciudad de Tunja. (Tesis de maestría), Universidad Pedagógica y Tecnológica de Colombia, Tunja.

Chaux, E. (2012). Educación, convivencia y agresión escolar. Bogota: Aguilar, Altea, Taurus, Alfaguara, S. A.

Consejo Superior de la Judicatura. (1991). Constitución Política de Colombia [Const.]. Recuperado de: http://www.corteconstitucional.gov.co/inicio/ Constitucion \%20politica\%20de\%20Colombia\%20-0202015.pdf.

Delgado-Salazar, R., \& Lara Salcedo, L. M. (2008). De la mediación del conflicto escolar. Bogotá: Pontificia Universidad Javeriana.

De Zubiría Samper, J. (2013). El maestro y los desafíos a la educación en el siglo XXI. Redipe Virtual, 825.

Duarte, J. D. (2005). Comunicación y convivencia escolar en la ciudad de Medellín, Colombia. Revista Iberoamericana de Educación, 3, 135-154.

Elliot, J. (1994). La investigación - acción en educación. Madrid: Morata.

Freire, P. (1970). Pedagogía del oprimido. Nueva York: Herder \& Herder.

Freire, P. (1993). Pedagogía de la Esperanza. México: Siglo XX.

García-Sánchez, B. Y., \& Ortiz Molina, B. I. (2012). Los maestros ante la violencia escolar. Bogotá: CADE.

Goleman, D. (1997). La inteligencia emocional. Buenos Aires: Vergara.

Lara-Salcedo, R. D. (2008). De la mediación de conflictos a comunidades más justas. Pontificia Universidad Javeriana, Bogotá, Colombia.

Laing. R. D. (1961). El Yo y los Otros. Ciudad de México: Fondo de Cultura Económica.

Maturana, H. \& Nisis, S. (1997). Formación bumana y capacitación. Santiago: Dolmen

\section{Ediciones.}

Maturana, H. (2008). El sentido de lo bumano. Santiago de Chile: Océano.

Mendoza, K. H., \& Rico Ballesteros, R. (2014). El clima escolar como elemento fundamental de la convivencia en la escuela. Escenarios, 12 (2), 7-18. 
Ministerio de Educación Nacional de Colombia. (8 de febrero de 1994). Ley General de Educación en Colombia [Ley 115 de 1994]. Recuperado de: http://www.mineducacion.gov.co/1621/articles-85906_archivo_pdf.pdf

Serna, C. M. (2014). Competencia socio afectiva en el marco escolar colombiano. Escenarios, 12 (2), 19-34. 